\title{
Clinical outcome in patients with hand lesions associated with complex regional pain syndrome after arthroscopic rotator cuff repair
}

\author{
Takaki Imai ${ }^{1}$, Masafumi Gotoh $^{2}$, Keiji Fukuda ${ }^{3}$, Misa Ogino $^{3}$, Hidehiro Nakamura ${ }^{2}$, Hiroki Ohzono ${ }^{4}$, \\ Naoto Shiba ${ }^{4}$, Takahiro Okawa ${ }^{2}$ \\ ${ }^{1}$ Department of Rehabilitation, Kyushu University of Nursing and Social Welfare, Kumamoto, Japan \\ ${ }^{2}$ Department of Orthopedic Surgery, Kurume University Medical Center, Fukuoka, Japan \\ ${ }^{3}$ Department of Orthopedic Surgery, Keishinkai Hospital, Saga, Japan \\ ${ }^{4}$ Department of Orthopedic Surgery, Kurume University, Kurume, Japan
}

Background: Complex regional pain syndrome (CRPS)-related hand lesions are one of the complications following arthroscopic rotator cuff repair (ARCR). This study aimed to investigate the clinical outcomes of patients with CRPS-related hand lesions following ARCR.

Methods: Altogether, 103 patients with ARCR were included in this study (mean age, 63.6 \pm 8.2 years; 66 males and 37 females; follow-up period, preoperative to 12 months postoperative). Clinical assessment included the Japanese Orthopaedic Association (JOA) score, University of California, Los Angeles (UCLA) score, Constant score, 36-item short form health survey (SF-36) score, and Quick Disabilities of the Arm, Shoulder, and Hand (QuickDASH) score from preoperative to 12 months postoperatively. The patients were either assigned to the CRPS group or non-CRPS group depending on CRPS diagnosis until the final follow-up, and clinical outcomes were then compared between the groups.

Results: Of 103 patients, 20 (19.4\%) had CRPS-related hand lesions that developed entirely within 2 months postoperatively. Both groups showed significant improvement in JOA, UCLA, and Constant scores preoperatively to 12 months postoperatively ( $\mathrm{p}<0.001)$. Comparisons between the two groups were not significantly different, except for SF-36 "general health perception" ( $\mathrm{p}<0.05)$ at 12 months postoperatively. At final follow-up, three patients had residual CRPS-related hand lesions with limited range of motion and finger edema.

Conclusions: CRPS-related hand lesions developed in 19.4\% of patients following ARCR. Shoulder or upper-limb function improved in most cases at 12 months, with satisfactory SF-36 patient-based evaluation results. Patients with residual CRPS-related hand lesions at the last follow-up require long-term follow-up.

Keywords: Shoulder; Rotator cuff injuries; Complex regional pain syndrome; Treatment outcome

\section{INTRODUCTION}

Rotator cuff tears commonly develop in middle-aged and elderly individuals [1]. Arthroscopic rotator cuff repair (ARCR) is a treatment option for patients with cuff tears [2,3] and is comparable in clinical outcome to open repair $[4,5]$. Additionally, the

Received: February 8, $2021 \quad$ Revised: March 15, $2021 \quad$ Accepted: March 18, 2021

Correspondence to: Takaki Imai

Department of Rehabilitation, Kyushu University of Nursing and Social Welfare, 888 Tominoo, Tamana Kumamoto 865-0062, Japan

Tel: +81-968-75-1800, Fax: +81-968-75-1811, E-mail: t-imai@kyushu-ns.ac.jp, ORCID: https://orcid.org/0000-0002-4399-2623

Financial support: None.

Conflict of interest: None.

Copyright@ 2021 Korean Shoulder and Elbow Society.

This is an Open Access article distributed under the terms of the Creative Commons Attribution Non-Commercial License (http://creativecommons.org/licenses/by-nc/4.0/) which permits unrestricted non-commercial use, distribution, and reproduction in any medium, provided the original work is properly cited. 
risk of postoperative complications is low due to decreased incisions in the skin and soft tissues [6]. However, vascular and neurologic injury, fluid extravasation, stiffness, and iatrogenic tendon injury may develop postoperatively [6,7].

Complex regional pain syndrome (CRPS) has various etiologic factors, including minor traumas, fractures, sprains, immobilization, and surgical interventions [8,9]. CRPS-related hand lesions are also a complication after ARCR, leading to atrophic changes, range of motion (ROM) limitation, hyperalgesia, paridrosis, and edema $[10,11]$. The incidence of CRPS-related hand lesions after ARCR is $11.0 \%-24.4 \%$ in the Japanese literature, which typically develops within 3 months postoperatively [12-16]. The reported incidence rate of CRPS varies according to the diagnostic criteria used, with an incidence rate of $24.2 \%$ based on the Ministry of Health, Labour and Welfare CRPS study team in the Japan (MHLWJ) evaluation system for "clinical purposes," $11 \%$ in the MHLWJ evaluation system for "research purposes," 6\% in the International Association for the Study of Pain (IASP) 2005 for "clinical purposes," and 0.5\% in the IASP 2005 for "research purposes"[11].

It has been reported in Japanese literature that CRPS-related hand lesions do not affect postoperative outcomes after ARCR $[13,14]$; however, a few English studies have been evaluated regarding these outcomes. Therefore, the present study aimed to investigate the clinical outcomes of patients with CRPS-related hand lesions after ARCR in a retrospective manner. We hypothesized that upper extremity function, including the shoulder joint, would not be affected by CRPS-related hand lesions after ARCR.

\section{METHODS}

The Institutional Review Board of Kurume University approved the study protocol (IRB No. 18129), and all subjects provided informed consent for participation.

\section{Subjects}

Between January 2014 and September 2017, 276 patients underwent ARCR for a rotator cuff tear at our institution. Of these, 158 patients were transferred to our hospital in the early postoperative period (within 3 weeks) for postoperative rehabilitation. The inclusion criteria were (1) individuals who had ARCR, (2) individuals who underwent a postoperative rehabilitation program and were available for follow-up for at least 1 year postoperatively, and (3) individuals who had rigorous imaging evaluation with magnetic resonance imaging (MRI). The exclusion criteria were (1) individuals with periarticular fracture, (2) individuals with progressive arthritis, (3) individuals with osteoarthritis, (4) indi- viduals with infection, (5) individuals with reoperation, and (6) individuals who had preoperative hand lesions such as ROM restriction or edema. Finally, 103 patients (66 males and $37 \mathrm{fe}$ males; mean age, $63.6 \pm 8.2$ years; mean period from onset to surgery, $10.4 \pm 11.6$ months) were included in this study (Table 1). Patient clinical scores and MRI images were extracted retrospectively from the medical records and imaging data.

\section{Surgical Procedure}

ARCR was considered for patients who did not respond to nonoperative treatment for $\geq 3$ months, which included the administration of anti-inflammatory medication, subacromial/glenohumeral injections of corticosteroids or hyaluronic acid, and rehabilitation with a focus on physical therapy. ARCR was performed with the patient in the beach chair position under general anesthesia. The torn cuff was repaired using a single-row (one row of anchors placed on the lateral aspect of the footprint and the torn cuff fixed with interrupted sutures) or suture bridge (one row of anchors placed on the medial aspect of the footprint with or without tying and the torn cuff fixed with a transosseous knotless anchor on the lateral aspect of the footprint) method. Acromioplasty was performed in all patients, and capsular release and biceps tendon procedures (tenotomy/tenodesis) were performed as needed.

\section{Postoperative Regimen}

Postoperatively, patients were immobilized in a sling for 6 weeks using an abduction pillow and were instructed to maintain the shoulder at neutral rotation and $20^{\circ}$ of abduction. Passive ROM exercises for the scapulothoracic, elbow, wrist, and finger joints were initiated immediately after surgery. Passive ROM of the glenohumeral joint was initiated 4 days postoperatively in small/ medium tears and 4 weeks postoperatively in large/massive tears. Active ROM exercise was permitted at 6 weeks postoperatively. At 8 weeks, isometric muscle strengthening exercises were introduced and at 12 weeks, isotonic muscle strengthening exercises were allowed.

\section{Diagnosis of CRPS}

The present study used the criteria suggested by the MHLWJ [8]. The hand was evaluated for five items (trophic changes, motor dysfunctions, abnormal sensory processing, asymmetric sudomotor activity, and asymmetric edema), and any applicable items were scored as 1 point, which were converted to a CRPS score of 0-5 points. For example, limited finger ROM was assigned 1 point for the motor dysfunctions item. CRPS was determined when at least two corresponding items were met subjectively and 
Table 1. Patient demographics

\begin{tabular}{|c|c|c|c|c|}
\hline Variable & Total $(n=103)$ & CRPS $(n=20)$ & Non-CRPS $(\mathrm{n}=83)$ & $\mathrm{p}$-value \\
\hline \multicolumn{5}{|l|}{ Demographic variable } \\
\hline Age (yr) & $63.6 \pm 8.2$ & $64.9 \pm 7.5$ & $63.3 \pm 8.4$ & $0.43^{\dagger}$ \\
\hline Sex (male:female) & $66: 37$ & $16: 4$ & $50: 33$ & 0.10 \\
\hline Diabetes & $12(11.7)$ & $1(5.0)$ & $11(13.3)$ & 0.30 \\
\hline Dominant-side surgery & $64(62.1)$ & $16(80.0)$ & $48(57.8)$ & $0.07^{*}$ \\
\hline Traumatic onset & $48(46.6)$ & $11(55.0)$ & $37(44.6)$ & $0.43^{*}$ \\
\hline Symptom duration (mo) & $10.4 \pm 11.6$ & $10.3 \pm 11.0$ & $10.4 \pm 11.8$ & $0.84^{\dagger}$ \\
\hline Workers' compensation & $14(13.6)$ & $2(10.0)$ & $12(14.5)$ & $0.60^{\ddagger}$ \\
\hline \multicolumn{5}{|l|}{ Structural variable } \\
\hline Tear size $(\mathrm{cm})$ & $2.6 \pm 1.2$ & $2.8 \pm 1.2$ & $2.5 \pm 1.2$ & $0.25^{\dagger}$ \\
\hline Tear size classification & & & & $0.67^{\ddagger}$ \\
\hline Small & 17 & 2 & 15 & \\
\hline Medium & 40 & 7 & 33 & \\
\hline Large & 40 & 10 & 30 & \\
\hline Massive & 6 & 1 & 5 & \\
\hline Preoperative global fatty degeneration index & $1.2 \pm 0.6$ & $1.1 \pm 0.7$ & $1.2 \pm 0.6$ & $0.48^{\dagger}$ \\
\hline Retear at 12 months & $14(13.6)$ & $4(20.0)$ & $10(12.0)$ & $0.40^{\ddagger}$ \\
\hline \multicolumn{5}{|l|}{ Intraoperative variable } \\
\hline Repair technique & & & & $0.77^{\ddagger}$ \\
\hline Suture bridge & 99 & 19 & 80 & \\
\hline Single row & 4 & 1 & 3 & \\
\hline Capsular release & $24(23.3)$ & $6(30.0)$ & $18(21.7)$ & $0.33^{\ddagger}$ \\
\hline Manipulation & $17(16.5)$ & $6(30.0)$ & $11(13.3)$ & $0.07^{\ddagger}$ \\
\hline Contracture & $24(23.3)$ & $7(35.0)$ & $17(20.5)$ & $0.18^{*}$ \\
\hline Treatment of long head of biceps tendon & & & & $0.48^{*}$ \\
\hline Untreated & $27(26.2)$ & $4(20.0)$ & $23(27.7)$ & \\
\hline Treated & $25(24.3)$ & $16(80.0)$ & $60(72.3)$ & \\
\hline
\end{tabular}

Values are presented as mean \pm standard deviation or number $(\%)$.

CRPS: complex regional pain syndrome.

${ }^{\star}$ Contracture was judged by manipulation or capsular release; ${ }^{\dagger}$ Continuous data: Wilcoxon rank sum test; ${ }^{\ddagger}$ Categorical data: chi-square test.

objectively in the MHLWJ "clinical objectives" evaluation system. A well-trained orthopedist blinded to the study diagnosed CRPS using these criteria. CRPS was assessed weekly from the immediate postoperative period until 12 weeks postoperatively and then at 3,6,9, and 12 months postoperatively.

Neurotropin (Nippon Zoki Pharmaceutical Co., Osaka, Japan) was administered orally to patients diagnosed with CRPS according to the CRPS criteria. If necessary, tramadol or pregabalin or both tramadol and pregabalin were added. Stellate ganglion block and vortex flow baths with laser beams were routinely applied. Patients with persistent symptoms were referred to an anesthesiologist who specializes in nerve blocks.

\section{Outcome Measures}

Patient information, including age, sex, and disease duration, was collected preoperatively. Japanese Orthopaedic Association (JOA) score (total score of 100 points, scored for items like pain, function, ROM, radiographic findings, and joint stability), University of California, Los Angeles (UCLA) score, Constant score, 36-item short form health survey (SF-36) score, and Quick Disabilities of the Arm, Shoulder, and Hand (QuickDASH) score were evaluated preoperatively and at 12 months postoperatively. Clinical outcomes of the study were assessed by three physiotherapists with more than 10 years of experience.

The integrity of the rotator cuff was determined using MRI at 12 months postoperatively. Fatty degeneration was assessed using the Goutallier classification (supraspinatus, infraspinatus, and subscapularis) on preoperative MRI "Y view" [16], and the global fatty degeneration index [17] was then calculated. The MRI data were evaluated by a radiologist familiar with orthopedic diseases.

\section{Statistical Analysis}

JMP 13 (SAS Institute Inc., Cary, NC, USA) was the software program used for statistical analysis. The Wilcoxon rank-sum 
test was used for continuous data such as age and clinical outcomes, and the chi-square test was used for categorical data such as sex and retear rate, to compare basic characteristics between the two groups. The Friedman test was used to compare the variance between CRPS scores from onset to 1 year postoperatively in the CRPS group. The Steel-Dwass test was used for multiple comparisons of CRPS scores. The significance level was set at $<5 \%$.

\section{RESULTS}

Of 103 patients, 20 (19.4\%) had CRPS-related hand lesions that developed entirely within 2 months postoperatively (mean $19.6 \pm 19.6$ days after surgery). Consequently, patients were divided into two groups: 83 patients in the non-CRPS group and 20 patients in the CRPS group. The CRPS score of the CRPS group was $2.4 \pm 0.5$ points at the onset of CRPS (range, 1-8 weeks), $2.05 \pm 0.67$ points at 8 weeks postoperatively, $2.05 \pm 0.76$ points at 9 weeks postoperatively, $1.95 \pm 0.83$ points at 10 weeks postoperatively, $1.9 \pm 0.85$ points at 11 weeks postoperatively, $1.8 \pm 0.89$ points at 12 weeks postoperatively, $0.95 \pm 1.0$ points at 6 months postoperatively, $0.5 \pm 0.76$ points at 9 months postoperatively, and $0.4 \pm 0.75$ points at 12 months postoperatively. There was a significant improvement at 6 months postoperatively compared to the onset of CRPS ( $p<0.001$ ) (Fig. 1). Symptoms at the onset of CRPS-related hand lesions included motor dysfunction of the fingers (100\%), edema (95\%), abnormal sensory processing (30\%), asymmetric sudomotor activity (15\%), and trophic changes (0\%).

The JOA scores (CRPS and non-CRPS group) were $65.3 \pm 12.4$ and $69.1 \pm 12.5$ preoperatively and $87.3 \pm 7.1$ and $88.0 \pm 9.4$ at 12

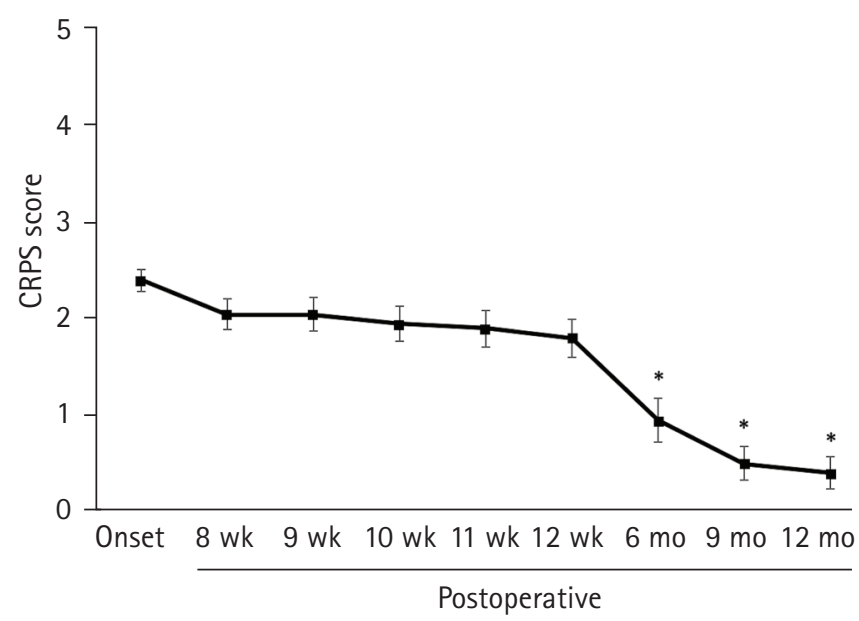

Fig. 1. Change in complex regional pain syndrome (CRPS) score from the onset of CRPS-related hand lesions to 12 months after surgery. ${ }^{*} \mathrm{P}<0.001$. months postoperatively, and both groups showed significant improvement at 12 months postoperatively compared with that observed preoperatively ( $\mathrm{p}<0.001$, respectively), but there was no significant difference between the two groups preoperatively and postoperatively (Fig. 2A). The UCLA scores (CRPS and nonCRPS group) were $15.6 \pm 5.1$ and $15.8 \pm 4.9$ preoperatively and $27.4 \pm 5.4$ and $29.2 \pm 5.8$ at 12 months postoperatively, and both groups showed significant improvement at 12 months postoperatively compared with that observed preoperatively $(\mathrm{p}<0.001)$, but there was no significant difference between the two groups preoperatively and postoperatively (Fig. 2B). The Constant scores (CRPS and non-CRPS group) were $47.8 \pm 17.8$ and $52.6 \pm 16.2$ preoperatively and $79.6 \pm 12.9$ and $80.0 \pm 14.9$ at 12 months postoperatively, and both groups showed significant improvement at 12 months postoperatively compared with that preoperatively $(\mathrm{p}<0.001)$, but there was no significant difference between the two groups preoperatively and postoperatively (Fig. 2C).

In the SF-36 scores, there were no significant differences between the two groups, except for "general health perceptions" at
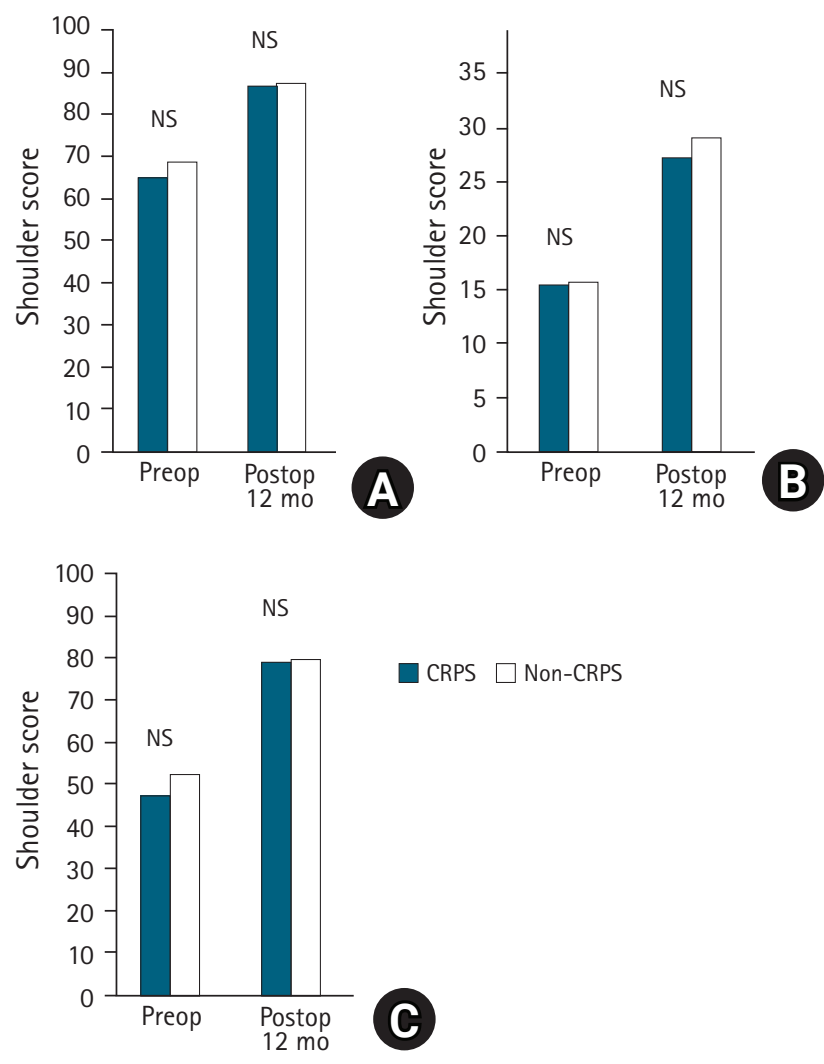

Fig. 2. Comparison of shoulder scores. (A) Japanese Orthopaedic Association score, (B) University of California at Los Angeles score, (C) Constant score. There were no statistically significant differences between the complex regional pain syndrome (CRPS) and nonCRPS groups in clinical shoulder scores before and at 12 months after surgery. NS: not significant. 
Table 2. Clinical results of SF-36 scores with or without CRPS

\begin{tabular}{lccc}
\hline Variable & $\begin{array}{c}\text { CRPS } \\
(\mathrm{n}=20)\end{array}$ & $\begin{array}{c}\text { Non-CRPS } \\
(\mathrm{n}=83)\end{array}$ & p-value \\
\hline Preoperative & & & \\
$\quad$ Physical functioning & $64.4 \pm 26.7$ & $75.0 \pm 16.3$ & 0.17 \\
Role physical & $51.8 \pm 28.8$ & $53.0 \pm 24.8$ & 0.86 \\
Bodily pain & $37.7 \pm 17.9$ & $43.1 \pm 19.6$ & 0.28 \\
General health perception & $56.4 \pm 16.1$ & $61.4 \pm 17.8$ & 0.28 \\
Vitality & $53.1 \pm 18.0$ & $57.7 \pm 19.7$ & 0.49 \\
Social functioning & $53.1 \pm 15.2$ & $65.3 \pm 24.5$ & 0.11 \\
Role emotional & $50.9 \pm 28.4$ & $62.0 \pm 30.5$ & 0.25 \\
Mental health & $53.7 \pm 23.9$ & $66.5 \pm 19.0$ & 0.07 \\
Postoperative 12 months & & & \\
Physical functioning & $77.0 \pm 24.6$ & $83.4 \pm 17.8$ & 0.29 \\
Role physical & $72.8 \pm 21.9$ & $77.3 \pm 23.7$ & 0.32 \\
Bodily pain & $68.1 \pm 17.8$ & $69.7 \pm 19.3$ & 0.85 \\
General health perception & $59.0 \pm 20.3$ & $67.3 \pm 16.7$ & $0.04^{*}$ \\
Vitality & $66.3 \pm 21.6$ & $67.7 \pm 17.5$ & 0.96 \\
Social functioning & $77.5 \pm 26.2$ & $85.4 \pm 20.4$ & 0.26 \\
Role emotional & $82.9 \pm 21.9$ & $82.3 \pm 22.7$ & 0.89 \\
Mental health & $73.5 \pm 20.7$ & $74.3 \pm 17.5$ & 0.76 \\
\hline
\end{tabular}

Values are presented as mean \pm standard deviation.

SF-36: 36-item short form health survey, CRPS: complex regional pain syndrome.

*Statistically significant among the two groups with the Wilcoxon ranksum test.

Table 3. Clinical results of QuickDASH scores with or without CRPS

\begin{tabular}{lccc}
\hline Variable & $\begin{array}{c}\text { CRPS } \\
(\mathrm{n}=20)\end{array}$ & $\begin{array}{c}\text { Non-CRPS } \\
(\mathrm{n}=83)\end{array}$ & p-value \\
\hline $\begin{array}{l}\text { Preoperative } \\
\quad \text { Disability/symptom }\end{array}$ & $38.2 \pm 18.6$ & $34.6 \pm 17.3$ & 0.39 \\
$\quad$ Work $(14 / 51)^{*}$ & $42.0 \pm 26.0$ & $43.0 \pm 30.6$ & 0.81 \\
$\quad$ Sports/music $(7 / 25)^{*}$ & $74.1 \pm 29.2$ & $53.3 \pm 38.8$ & 0.18 \\
Postoperative 12 months & & & \\
$\quad$ Disability/symptom & $14.8 \pm 10.7$ & $12.7 \pm 13.9$ & 0.15 \\
$\quad$ Work $(13 / 52)^{*}$ & $14.4 \pm 14.3$ & $13.9 \pm 18.5$ & 0.68 \\
$\quad$ Sports/music $(9 / 36)^{*}$ & $24.3 \pm 31.5$ & $18.2 \pm 22.4$ & 0.97 \\
\hline
\end{tabular}

Values are presented as mean \pm standard deviation.

QuickDASH: Quick Disabilities of the Arm, Shoulder, and Hand, CRPS: complex regional pain syndrome.

${ }^{*}$ Number of respondents (CRPS/non-CRPS).

12 months postoperatively $(\mathrm{p}<0.05)$ (Table 2). In the QuickDASH scores, there were no significant differences between the two groups in all items preoperatively and 12 months postoperatively (Table 3).

At the final follow-up, three patients had residual CRPS-related hand lesions with limited ROM and edema of the fingers. These three patients showed a CRPS score of 2 points at the final follow-up, whereas the CRPS score was $>3$ points at the onset. The clinical scores of these three patients were relatively low compared with those of other groups (17 patients in the CRPS group and 83 patients in the non-CRPS groups) (Table 4).

\section{DISCUSSION}

Complications associated with ARCR, including loose hardware, failure of repair, traction in the lateral position, direct injury, compression secondary to fluid extravasation, tourniquet-like problems associated with wrapping the operative extremity, and postoperative stiffness have been reported [6]. CRPS-related hand lesions also develop after ARCR although there has been a paucity of English literature that has focused on this complication. In several Japanese reports, CRPS incidence was reported to be $21.7 \%, 11.8 \%$, and $16.2 \%[14,18,19]$ according to the MHLWJ criteria. Thus, CRPS-related hand lesions develop mainly on the surgical side within 3 months postoperatively, and similar results were observed in this study. However, it has been reported that the development of hand lesions related to CRPS may be triggered by exacerbation of shoulder pain due to orthotic removal $[14,15]$. In this study, the abduction brace was removed at 6 weeks postoperatively, but the timing of brace removal may be affected.

Previous Japanese studies have shown that CRPS-related hand lesions do not affect shoulder function after ARCR [13-15]. Kobayashi et al. [15] compared the JOA scores at 1 year postoperatively in the early-onset group with those in the late-onset group and non-CRPS group. As a result, the period from onset did not affect the clinical outcome of patients in these groups. Kiba et al. [18] compared the JOA scores at 6 months postoperatively in the presence or absence of symptoms. In their study, CRPS-related hand lesions had no effect on the clinical outcomes; however, these studies focused only on shoulder function and not on the whole upper-limb function. The present study evaluated both functions and found that coexisting CRPS-related hand lesions have less effect on whole upper-limb function, including shoulder function.

Generally, CRPS develops as a hand lesion on the operative side following ARCR [9-15,18,19]. Kobayashi et al. [14] found that all symptoms improved in an average of 7 months following ARCR. In a study by Kiba et al. [18], CRPS-related hand lesions improved in $80 \%$ of the cases by 6 months postoperatively, but this symptom was persistent in a case with high CRPS score of $\geq 3$ points at onset. In the present study, CRPS-related hand lesions improved in $75 \%$ of cases at 6 months and in $85 \%$ of cases at 9 months postoperatively; however, three patients with a CRPS score $\geq 3$ points still had residual ROM restriction of the finger and/or extensive edema on the dorsal side at 12 months postop- 
Table 4. Clinical scores for patients with CRPS-unimproved, CRPS-improved, and non-CRPS groups

\begin{tabular}{|c|c|c|c|c|}
\hline \multirow{2}{*}{ Variable } & & \multicolumn{2}{|c|}{ CRPS $(n=20)$} & \multirow{2}{*}{ Non-CRPS $(\mathrm{n}=83$} \\
\hline & & Unimproved $(n=3)$ & Improved $(n=17)$ & \\
\hline \multirow[t]{2}{*}{ JOA score } & Preop & $56.8 \pm 10.8$ & $66.7 \pm 12.3$ & $69.1 \pm 12.5$ \\
\hline & Postop $12 \mathrm{mo}$ & $83.5 \pm 8.2$ & $87.9 \pm 5.8$ & $88.0 \pm 9.4$ \\
\hline \multirow[t]{2}{*}{ UCLA score } & Preop & $16.0 \pm 4.6$ & $15.5 \pm 5.3$ & $15.8 \pm 4.9$ \\
\hline & Postop $12 \mathrm{mo}$ & $25.7 \pm 14.4$ & $27.7 \pm 5.6$ & $29.2 \pm 5.8$ \\
\hline \multirow[t]{2}{*}{ Constant score } & Preop & $41.0 \pm 20.2$ & $49.1 \pm 17.7$ & $52.6 \pm 16.2$ \\
\hline & Postop $12 \mathrm{mo}$ & $69.0 \pm 14.0$ & $81.4 \pm 12.2$ & $80.0 \pm 14.9$ \\
\hline \multicolumn{5}{|l|}{ QuickDASH } \\
\hline \multirow[t]{2}{*}{ Disability/symptom } & Preop & $55.7 \pm 20.9$ & $35.7 \pm 17.7$ & $34.6 \pm 17.3$ \\
\hline & Postop 12 mo & $25.0 \pm 6.0$ & $13.0 \pm 10.4$ & $12.7 \pm 13.9$ \\
\hline \multirow[t]{2}{*}{ Work } & Preop & - & $42.0 \pm 26.0$ & $43.0 \pm 30.6$ \\
\hline & Postop 12 mo & $31.3 \pm 8.8$ & $11.4 \pm 13.1$ & $13.9 \pm 18.5$ \\
\hline \multirow[t]{2}{*}{ Sports/music } & Preop & 100 & $69.8 \pm 29.4$ & $53.3 \pm 38.8$ \\
\hline & Postop 12 mo & $46.9 \pm 39.8$ & $17.9 \pm 29.0$ & $18.2 \pm 22.4$ \\
\hline \multicolumn{5}{|l|}{ SF-36 } \\
\hline \multirow[t]{2}{*}{ Physical functioning } & Preop & $35.0 \pm 14.1$ & $70.3 \pm 23.5$ & $75.0 \pm 16.3$ \\
\hline & Postop $12 \mathrm{mo}$ & $36.7 \pm 24.7$ & $84.1 \pm 16.8$ & $83.4 \pm 17.8$ \\
\hline \multirow[t]{2}{*}{ Role physical } & Preop & $21.9 \pm 4.4$ & $57.8 \pm 27.8$ & $53.0 \pm 24.8$ \\
\hline & Postop $12 \mathrm{mo}$ & $60.4 \pm 25.3$ & $75.0 \pm 21.3$ & $77.3 \pm 23.7$ \\
\hline \multirow[t]{2}{*}{ Bodily pain } & Preop & $26.5 \pm 6.4$ & $39.9 \pm 18.9$ & $43.1 \pm 19.6$ \\
\hline & Postop 12 mo & $62.0 \pm 21.5$ & $69.2 \pm 17.6$ & $69.7 \pm 19.3$ \\
\hline \multirow[t]{2}{*}{ General health perception } & Preop & $42.5 \pm 3.5$ & $59.2 \pm 16.3$ & $61.4 \pm 17.8$ \\
\hline & Postop $12 \mathrm{mo}$ & $41.3 \pm 9.3$ & $62.1 \pm 20.2$ & $67.3 \pm 16.7$ \\
\hline \multirow[t]{2}{*}{ Vitality } & Preop & $34.4 \pm 13.3$ & $56.9 \pm 16.8$ & $57.7 \pm 19.7$ \\
\hline & Postop $12 \mathrm{mo}$ & $62.5 \pm 25.0$ & $66.9 \pm 21.7$ & $67.7 \pm 17.5$ \\
\hline \multirow[t]{2}{*}{ Social functioning } & Preop & $37.5 \pm 0$ & $56.3 \pm 14.7$ & $65.3 \pm 24.5$ \\
\hline & Postop $12 \mathrm{mo}$ & $58.3 \pm 14.4$ & $80.9 \pm 26.6$ & $85.4 \pm 20.4$ \\
\hline \multirow[t]{2}{*}{ Role emotional } & Preop & $16.7 \pm 11.8$ & $57.8 \pm 25.6$ & $62.0 \pm 30.5$ \\
\hline & Postop 12 mo & $80.6 \pm 17.3$ & $83.3 \pm 23.0$ & $82.3 \pm 22.7$ \\
\hline \multirow[t]{2}{*}{ Mental health } & Preop & $45.0 \pm 21.2$ & $55.5 \pm 25.1$ & $66.5 \pm 19.0$ \\
\hline & Postop 12 mo & $46.7 \pm 20.2$ & $78.2 \pm 17.3$ & $74.3 \pm 17.5$ \\
\hline
\end{tabular}

Values are presented as mean \pm standard deviation.

CRPS: complex regional pain syndrome, JOA: Japanese Orthopaedic Association, Preop: preoperative, UCLA: University of California, Los Angeles, QuickDASH: Quick Disabilities of the Arm, Shoulder, and Hand, SF-36: 36-item short form health survey, Preop: preoperative, Postop: postoperative.

eratively. de Mos et al. [20] reported that the degree of CRPS-related hand lesions was associated with remission periods; thus, hand lesions in cases with a high CRPS score ( $\geq 3$ points) at onset may be persistent and further long-term follow-up will be required in these patients.

In 1994, the IASP criteria were proposed as a diagnostic method for CRPS [21,22] with high sensitivity and low specificity [23], which was then revised in 2005 [24]. To establish more specific and suitable criteria for the Japanese population, an original diagnostic criterion was developed in 2010 by the MHLWJ research team (sensitivity, 0.83 and specificity, 0.79) [8]. The incidence of CRPS-related hand lesions following ARCR using this criterion ranged from $11.7 \%$ to $21.7 \%[14,18,19]$ compared with that using the IASP 2005 criteria (ranging from $0.4 \%$ to 6\%) [9-11]. It has been suggested that the IASP diagnostic criteria are affected by different cultural backgrounds and health care systems [25], and that the MHLWJ criteria are more suitable for Japanese people [8]. This may explain why the incidence of CRPS was relatively higher using the MHLWJ criteria than the IASP 2005 criteria. However, the low incidence of IASP criteria confirms another diagnosis with similar clinical manifestations and appropriate treatment.

Harada et al. [26,27] reported that the most common complication with hand symptoms after ARCR is flexor tenosynovitis 
followed by carpal tunnel syndrome, and prompt treatment with corticosteroid injections is recommended. Thus, the possibility that hand lesions after ARCR may be influenced by other diseases presenting with CRPS-like symptoms is becoming clearer. Bharwani et al. [28] stated that the pathophysiology of CRPS is multifactorial; therefore, there is a need for systematic diagnosis and treatment of CRPS symptoms with an algorithm. In view of these findings, a systematic diagnosis and treatment of hand lesions after ARCR using multiple criteria is recommended.

This study had several limitations. First, it has a retrospective design with a small sample size and short follow-up period. Second, the effects of drug treatments given only to the CRPS group cannot be ruled out. Third, all surgeries were performed in the beach chair position, and it is not clear whether differences in surgical posture affect hand symptoms. Fourth, the follow-up rate was relatively low because the subjects of the present study were patients transferred to our hospital for postoperative rehabilitation. However, the CRPS-related hand lesion incidence in this study was close to that observed in previous studies [11-16], and validity of this study was proven to some extent. As a strength of this study, we confirmed three cases in which symptoms of CRPS-related hand lesions persisted at the final follow-up, and these hand lesions did not essentially affect upper-limb function, including the involved shoulder.

CRPS-related hand lesions developed in $19.4 \%$ of patients after ARCR. Shoulder or upper-limb function improved in most cases at 12 months, with satisfactory results of SF-36 patient-based evaluation. ROM of the involved fingers did not improve in three patients with high CRPS score at onset; therefore, long-term follow-up will be necessary in these patients.

\section{ORCID}

Takaki Imai

https://orcid.org/0000-0002-4399-2623

\section{REFERENCES}

1. Kukkonen J, Joukainen A, Lehtinen J, et al. Treatment of nontraumatic rotator cuff tears: a randomized controlled trial with two years of clinical and imaging follow-up. J Bone Joint Surg Am 2015;97:1729-37.

2. Iyengar JJ, Samagh SP, Schairer W, Singh G, Valone FH 3rd, Feeley BT. Current trends in rotator cuff repair: surgical technique, setting, and cost. Arthroscopy 2014;30:284-8.

3. McElvany MD, McGoldrick E, Gee AO, Neradilek MB, Matsen FA 3rd. Rotator cuff repair: published evidence on factors associated with repair integrity and clinical outcome. Am J Sports

\section{Med 2015;43:491-500.}

4. Severud EL, Ruotolo C, Abbott DD, Nottage WM. All-arthroscopic versus mini-open rotator cuff repair: a long-term retrospective outcome comparison. Arthroscopy 2003;19:2348.

5. Morse K, Davis AD, Afra R, Kaye EK, Schepsis A, Voloshin I. Arthroscopic versus mini-open rotator cuff repair: a comprehensive review and meta-analysis. Am J Sports Med 2008;36: 1824-8.

6. Weber SC, Abrams JS, Nottage WM. Complications associated with arthroscopic shoulder surgery. Arthroscopy 2002;18(2 Suppl 1):88-95.

7. Owens BD, Williams AE, Wolf JM. Risk factors for surgical complications in rotator cuff repair in a veteran population. J Shoulder Elbow Surg 2015;24:1707-12.

8. Sumitani M, Shibata M, Sakaue G, Mashimo T; Japanese CRPS Research Group. Development of comprehensive diagnostic criteria for complex regional pain syndrome in the Japanese population. Pain 2010;150:243-9.

9. Brislin KJ, Field LD, Savoie FH 3rd. Complications after arthroscopic rotator cuff repair. Arthroscopy 2007;23:124-8.

10. McBirnie JM, Miniaci A, Miniaci SL. Arthroscopic repair of full-thickness rotator cuff tears using bioabsorbable tacks. Arthroscopy 2005;21:1421-7.

11. Tanesue R, Gotoh M, Mitsui Y, et al. Hand lesion after arthroscopic rotator cuff repair: association with complex regional pain syndrome. J Orthop Sci 2018;23:70-4.

12. Asakura T, Matsuura K, Shin K, Ooe K. CRPS type 1 after arthroscopic rotator cuff repair. Katakansetsu (Shoulder Jt) 2011; 35:547-59.

13. Harada M, Mura N, Tsuruta D, Takagi M. Complications in arthroscopic rotator cuff repair associated with outcome of the arthroscopic surgery. Katakansetsu (Shoulder Jt) 2014;38:912-5.

14. Kobayashi H, Hata Y, Ishigaki N, et al. CRPS type I after operation with rotator cuff tears. Katakansetsu (Shoulder Jt) 2010; 34:463-6.

15. Kobayashi H, Hata Y, Seki H, Murakami N, Katoh H. Reflex sympathetic dystrophy after surgery for rotator cuff tears. Katakansetsu (Shoulder Jt) 2004;28:549-52.

16. Goutallier D, Postel JM, Bernageau J, Lavau L, Voisin MC. Fatty muscle degeneration in cuff ruptures: pre- and postoperative evaluation by CT scan. Clin Orthop Relat Res 1994;(304):78-83.

17. Goutallier D, Postel JM, Gleyze P, Leguilloux P, Van Driessche S. Influence of cuff muscle fatty degeneration on anatomic and functional outcomes after simple suture of full-thickness tears. J Shoulder Elbow Surg 2003;12:550-4.

18. Kiba T, Morishita T, Tachiiri H, Kubo T, Kurokawa M. Clinical 
outcome of symptoms like CRPS after rotator cuff repair. Katakansetsu (Shoulder Jt) 2011;35:889-92.

19. Tachiiri H, Morihara T, Kiba T, Kubo T, Kurokawa M. Change in the hand after rotator cuff repair: relation with CRPS type I. Katakansetsu (Shoulder Jt) 2010;34:495-8.

20. de Mos M, Huygen FJ, van der Hoeven-Borgman M, Dieleman JP, Ch Stricker BH, Sturkenboom MC. Outcome of the complex regional pain syndrome. Clin J Pain 2009;25:590-7.

21. Boas R. Complex regional pain syndromes: symptoms, signs and differential diagnosis. In: Janig W, Stanton-Hicks M, eds. Reflex sympathtic dystrophy: diagnosis. Seattle, WA: IASP Press; 1996. p. 79-92.

22. Merskey H, Bogduk N. Classification of chronic pain: descriptions of chronic pain syndromes and definitions of pain terms. 2nd ed. Seattle, WA: IASP Press; 1994.

23. Galer BS, Bruehl S, Harden RN. IASP diagnostic criteria for complex regional pain syndrome: a preliminary empirical validation study. International Association for the Study of Pain.
Clin J Pain 1998;14:48-54.

24. Harden RN, Bruehl S, Stanton-Hicks M, Wilson PR. Proposed new diagnostic criteria for complex regional pain syndrome. Pain Med 2007;8:326-31.

25. Perez RS, Collins S, Marinus J, Zuurmond WW, de Lange JJ. Diagnostic criteria for CRPS I: differences between patient profiles using three different diagnostic sets. Eur J Pain 2007;11:895902.

26. Harada M, Mura N, Takahara M, Takagi M. Complications of the fingers and hand after arthroscopic rotator cuff repair. Open Orthop J 2018;12:134-40.

27. Harada M, Mura N, Takahara M, Tsuruta D, Takagi M. Early detection and treatment of complications in the fingers and hand after arthroscopic rotator cuff repair. JSES Int 2020;4:61218.

28. Bharwani KD, Dirckx M, Huygen FJ. Complex regional pain syndrome: diagnosis and treatment. BJA Educ 2017;17:262-8. 